\title{
Erratum to: Modeling the water balance of sloped vineyards under various climate change scenarios
}

Marco Hofmann and Hans R. Schultz

Hochschule Geisenheim University, Institut für allgemeinen und ökologischen Weinbau, Von-Lade-Str. 1, 65366

Geisenheim, Germany

Original article:

BIO Web of Conferences 5, 01026 (2015), DOI: 10.1051/bioconf/20150501026

1. The formula (1) page 2 should be:

$$
T_{0, v}=\frac{R_{v}}{R_{v y}} E T_{0} .
$$

2. The formula (2) page 2 should be:

$$
T_{a, v}=k_{c, v} k_{s} T_{0, v}
$$

